\title{
FORUM
}

\section{The Future of the Knot as a Unit of Speed}

\section{Oliver Stewart}

FEw will deny the merits of the knot as a unit of speed. It does in one syllable what all other units of speed take three or more to do. It is accepted and used by a great many countries, including those like France which show a general preference for the metric system. Aviation has taken to it as well as shipping. It is not therefore surprising that the full acceptance of the Systeme International d'Unités (or S.I.) is meeting with some opposition when it offers to supplant the knot.

At present the situation is that the knot is to continue for a 'limited time' as a speed unit for use in aviation and shipping. Just how limited the time will be has not been revealed, but the member countries of the European Economic Community have set I January 1978 as the date after which 'only a prescribed system of metric units may be used'. Strictly interpreted the S.I. admits one and only one speed unit, the metre per second; if the minute or the hour were to be introduced in place of the second, decimalization would break down. This would mean that the familiar kilometre per hour would be inadmissible as a substitute for the knot, although the present trend is in that direction.

Both the metre and the second are now defined in terms of atomic radiation with a precision far ahead of anything previously known and give the measurer the highest attainable accuracy. In the wording of the British Standards Institution the metre is: the length equal to $I, 650,763.73$ wavelengths in vacuum of the radiation corresponding to the transition between the levels $2 p_{10}$ and $5 d_{5}$ of the krypton-86 atom, while the second is: the duration of $9,192,631,770$ periods of the radiation corresponding to the transition between the two hyperfine levels of the ground state of the caesium-1 33 atom. These definitions are more complicated than the earlier platinum-iridium metre bar and the fraction of the tropical year, but the nautical mile and the hour, which are brought together in the knot, have less ultimate validity.

Originating as an angular measurement, the nautical mile was the best measure of distances on the surface of the sea, but purely linear definitions blur the relationship. There are also differences between the linear definitions; the British nautical mile is defined as $6080 \mathrm{ft}$. ( $1853.18 \mathrm{~m}$ ), the international nautical mile is exactly $1852 \mathrm{~m}$. The knot is one international nautical mile per hour, but to retain it would weaken the S.I. and the whole metrological reform now in progress.

Terminologically the metre per second is inferior to the knot. So are the kilometre per hour and the mile per hour. It is a pity that the S.I. has not introduced a special name for its speed unit, as for its pressure unit, the pascal (after Blaise Pascal the mathematician). If popular acceptance for the metre per second as a speed unit is looked for, there is a strong case for giving it a name. Perhaps it is not too fanciful to propose that the metre per second should be called the schneider, after Jacques Schneider who promoted the air race which had a power- 
ful influence in stimulating speed development. Since the international nautical mile is now defined in round figures in metres, users of the knot have already gone part of the way towards changing to the S.I., though possibly without knowing it. The second has a slightly less illustrious reputation and this has been emphasized by the devices that have had to be introduced in the radio time signals to make some sensible compromise between Greenwich Mean Time and the caesium second. The introduction of an internationally accepted speed unit of one metre per second deserves more favourable treatment than it has so far received.

EDITORIAL NOTE. It may be added that the new unit is very nearly equal to $2 \mathrm{kt}$ $(1 \cdot 944)$ and that it might also well be named after William Bourne who in 1574 first described the log and line, 'the first English contribution to the mariner's art' as Professor E. G. R. Taylor remarks. 